\title{
Using 360-Video Virtual Reality to Influence Caregiver Emotions and Behaviors for Childhood Literacy
}

\author{
Iulian Radu, Harvard University, USA \\ Chris Dede, Harvard University, USA \\ Mohamed Raouf Seyam, Harvard University, USA \\ iD https://orcid.org/0000-0002-8883-3255 \\ Tianyi Feng, Harvard University, USA \\ Michelle Chung, Harvard University, USA
}

\begin{abstract}
Through the design and exploratory evaluation of a narrative-based 360-video virtual reality experience, the authors aimed at building empathy in adults towards children who experience challenges in early literacy. This contributes to a limited literature on VR empathetic design by specifically studying caregivers in relation to reading difficulties and utilizing a low-cost immersive medium. This research performed a quasi-experimental pilot study following a pretest-posttest design with 27 participants, collecting measures such as participant empathy, anxiety, immersion, and emotional reactions. This paper explored changes in pre-post measures, correlations between variables, and possible explanations for the observed results. The VR experience increased positive caregiver attitudes towards struggling readers. Participants who reported a high degree of emotional reactions showed increased willingness to donate to help reading difficulties. Participants with teaching experience or with lower starting empathy scores were less likely to be affected.
\end{abstract}

\section{KEYWORDS}

360 Video, Dyslexia, Empathy, Immersion, Literacy, Presence, Virtual Reality, VR

\section{INTRODUCTION}

This study is part of an ongoing program of research that intends to identify what barriers are preventing adults from effectively building childhood early literacy, and subsequently to design experiences that can mitigate those factors via upskilling. One important barrier is the lack of empathy. Adults (parents, teachers, and other caregivers) who have high literacy often cannot relate to the experience of a struggling reader (Psychogiou et al. 2008; Gondoli \& Silverberg, 1997). They often do not identify with how hard it is when one is starting out reading, or what it feels like to have certain reading difficulties. Through narrative-based 360-video virtual reality experiences, we aim to build 
empathy in adults for the children in their lives, helping them to be more patient and understanding about the challenges of early literacy. This specific study involved constructing a pilot VR experience designed to induce emotions related to empathy, then studying its effects on caregiver participants who were parents and teachers. This experience is a first step towards creating larger-scale interventions designed to make participants more empathetic.

Scholars have studied the power of immersive media to aid with "unlearning": letting go of a routinely practiced, emotionally rooted identity to achieve transformational change to a different, more effective set of behaviors. Brook, Pedler, Abbott, and Burgoyne (2016) argue that the result of unlearning may be moving from knowing to not-knowing and from action to non-action, as a transitional step towards developing some transformed form of knowing and acting. Studies in virtual reality show that unlearning can be based on a series of powerful experiences that influence the mind/brain cognitively and affectively, intrapersonally and interpersonally (Bailenson, 2018; Slater $\&$ Sanchez-Vives, 2016). In particular, studies have documented the effectiveness of immersive authentic simulations for improving teacher effectiveness (Christiansen, Knezek, Tyler-Wood, \& Gibson, 2011; Seidel, Blomberg, \& Renkl, 2013; Badiee \& Kaufman, 2014; Straub, Dieker, Hynes, \& Hughes, 2014).

Empathy, the ability to detect, feel, and respond to another person's emotional states, is critical to developing social relationships. For parents, this ability can help sensitively guide parenting behaviors, especially in situations where children are not capable of communicating their internal emotional states (Fonagy, Gergely, \& Target, 2007). Research has shown that empathy can be developed through playing PC based video games (Bachen, Hernández-Ramos, \& Raphael, 2012; Greitemeyer, Osswald, \& Brauer, 2010), and recent work on 360 video narratives and VR shows that increased immersion can induce higher levels of empathy (Archer \& Finger, 2018; Shin, 2018). Mediums such as full-body-tracking VR can allow a user to embody another gender or race and have been shown to reduce racial bias (Peck et. al., 2013; Banakou, Hanumanthu, \& Slater, 2016) and to increase empathy towards users with physical disabilities (Väyrynen, Colley, \& Häkkilä, 2016). In VR studies where users embody a child, there are effects of distorted spatial perception (Banakou, Groten, \& Slater, 2013) and increases in empathy for situations where a parent negatively treats a child (Hamilton-Giachritsis, 2018). These are all forms of unlearning.

This study builds on this body of research and investigates whether factors that can influence caregivers' empathy can be increased by experiencing a typical situation in a struggling reader's life. Rather than full-body tracking VR immersion, this research uses the popular medium of 360 video, in a 3-degree-of-freedom VR experience (via Oculus Go head-mounted display). Establishing the effectiveness of a simpler and less expensive immersive medium than previous work is important in increasing scalability using lower-cost media and hardware. Studying the design of this alternative medium is crucial to ensuring its potential impact is realized.

\section{RELATED WORK}

Ongoing reciprocal social interactions between children and caregivers are critical components of child development, including early literacy development (Pellegrini \& Galda, 2003; Farrant \& Zubrick, 2012), which is a main predictor of academic success (Hoff, 2003). Caregivers who are attentive to their children provide sustained back-and-forth interactions which develop and shape children's mastery of language, by helping early readers to understand words they may be unfamiliar with, and to perform reading tasks they may be struggling with (Hirsh-Pasek et al., 2015; Rowe et al. 2017). The empathetic abilities of caregivers influence children's language and socioemotional development. As children observe and internalize the caregiver's tone of voice, touch, facial expressions, they learn to communicate and self regulate (Kohn, 1998). Research shows that parents who are more empathetic are more effective at perceiving their child's state and responding appropriately to child's needs (Psychogiou et al. 2008; Gondoli \& Silverberg, 1997). Based on this research, we hypothesize that 
being able to help a caregiver remember how it feels to be in a challenging situation as a child, has the potential to contribute to increased empathy and improved mentoring responses.

In a broad sense, immersive VR experiences have been demonstrated to be capable of impacting adult behaviors, thoughts and attitudes in a similar way to real-world experiences (Baily \& Bailenson, 2017). Typically these studies have been done using immersive virtual reality systems that track a user's movements and hands, allowing interactivity with the virtual environment. In one study, Formosa et al. (2018) tested adult reactions to becoming a person with schizophrenia through an interactive VR experience. The results showed that participants experience improvement in empathetic understanding towards people with schizophrenia. In a similar fashion, Dyer, Swartzlander and Gugliucci (2018) used VR immersion to improve medical students' empathy towards older patients, and reported that the VR experience intervention increased empathy of participants for older adults with agerelated health complications such as visual or hearing difficulties and Alzheimer's disease. Another experimentally controlled study on kindergarten teachers found that participants who went through 10 hours of simulation of being a virtually-simulated child in a VR subsequently demonstrated a better understanding of children's feelings, perceptions and needs, and exhibited higher levels of empathy towards the children compared to participants who went through 10 hours of workshop experience (Katz, 1999). In another study Hamilton-Giachritsis et al. (2018) created an immersive VR experience depicting a moment in the life of a 4-year old child, seen by the user from the perspective of the child. Mothers who participated in the study showed increased empathy when exposed to a virtual parent who expressed aggressive behaviors towards the child. Such VR experiences of being a victim of emotional abuse can cause observable changes in brain function, as shown in the study by Seinfeld et al. (2020). Participants who experienced a first-person VR episode of being a victim of emotional aggression showed enhanced emotion recognition abilities, that were observable through fMRI brain scans after the experience.

All this research shows that immersive virtual reality experiences have the potential to change participant empathy when having a first-person experience. However, such immersive experiences are expensive due to the hardware required to track a user's body and the costs required to build the virtual experience. In this research we are interested in understanding the design and effect of empathy-inducing experiences through lower-cost hardware, which is typically less immersive and thus may create a weaker impact.

With the increased availability of 360-degree cameras, it is now possible to create low-cost VR experiences by immersive the viewer in a prerecorded video. Such 360-video narratives have been created to depict challenging humanitarian crises and lead to increased viewer empathy, for example the United Nations film Clouds Over Sidra which follows a young girl living in a refugee camp (Schutte \& Stilinović, 2017). Among other studies of VR interventions that aim to improve caregiver empathy, one study conducted on caregivers of people with dementia found that caregiver empathy, confidence and positive interactions with those they cared for improved significantly after a 360-degree movie environment that allows the participant to take the perspective of a person with dementia (Wijma et al, 2017). In a similar depiction, (Adefila et al., 2016) showed that a 360-video narrative improved caregivers' empathy towards adults living with dementia.

While such 360-video experiences have been growing in popularity in recent years, there is a lack of research on caregivers' empathy towards people who have difficulties reading. Furthermore, there is a lack of research using 360-video experiences to depict the life of a child, likely due to the challenges of simulating a child's perspective through recorded videos. In this research we explore the possibilities of using a low-cost 360-video narrative to address these limitations.

\section{STUDY DESIGN}

Through narrative-based 360-video virtual reality experiences like those shown in Figure 1, this study aimed to increase emotional factors related to empathy in adults towards children who struggle 
Figure 1. The participant's view of the experience, showing the mother talking to the child (participant)

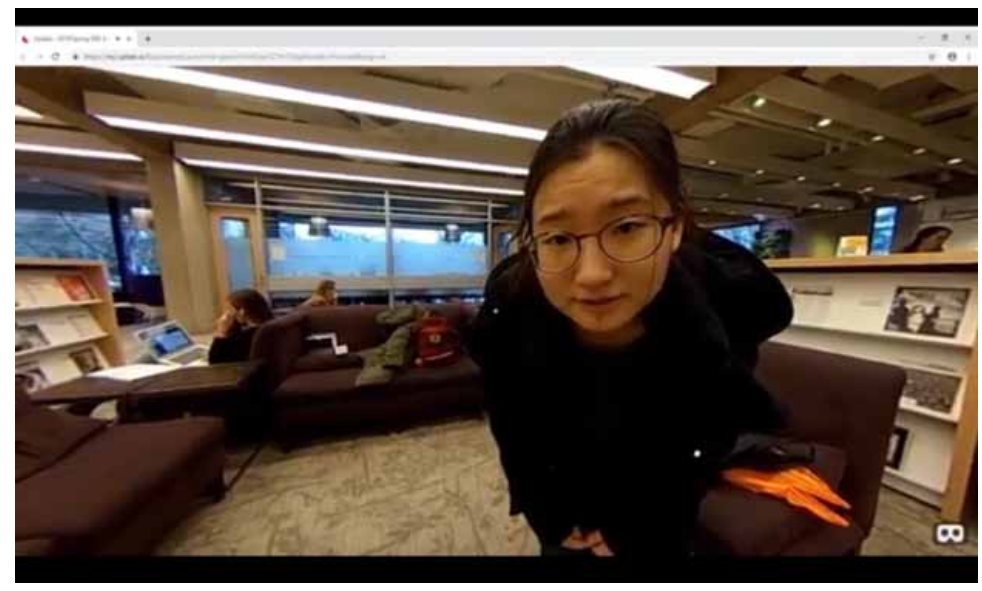

with reading, helping caregivers to be more patient and understanding toward the challenges of early literacy. The research questions are:

RQ1: What kind of emotional reaction does this experience create in the participants? How strong is the emotional reaction?

RQ2: To what degree does this experience change participant attitudes or behaviors towards struggling children?

RQ3: What factors of the experience influence participant emotions, attitude/behavior changes?

RQ4a: What design features enhanced participants' experiences?

RQ4b: What design features hindered participants' experiences?

In this study, we developed a 360-video VR experience that simulates the experience of being a child who suffers from difficulties reading. The experience is designed to stimulate multiple aspects of empathy, such as cognitive (increase user understanding by providing information about other situations influenced by reading difficulties, as well as information about proper parenting strategies), somatic (generate visceral reactions through features such as short perspective, heartbeats, background crying sounds), and emotional (generate compassionate reaction in participants by emphasizing inability to achieve tasks, present indicators that the child is increasingly distressed). The experience is introduced from the perspective of a narrator, who is an adult remembering a childhood memory in a bookstore.

As the experience starts, the participant is embodied in the role of the child (Figure 1), who wants to help their mother find a book for their sister's birthday. The narrative proceeds through several stages (Figure 2). The participant experiences all these stages through recorded 360-video segments as seen from the perspective of the child. Throughout the experience, participant agency is balanced by providing choices to interact (e.g., asking others for help, choosing books to examine), but is constrained by the overall sequence of the narrative. In the interactive segments, participant can click on hotspot areas of the 360-video to see additional recordings (such as clicking on a book and seeing it open) or to be moved to another chapter of the narrative.

The experience starts with an orientation outside the bookstore, intended to familiarize the participants with how to look around and navigate the experience. This introduction sequence ensures participant comfort before starting the main narrative. Once the participant moves inside the building, the mother is seen from the first-person perspective of the child (Figure 1) discussing the task with the 
Figure 2. Narrative sequence in three main parts: mother and child setting the task; child not finding the book due to inability to understand text and becoming anxious; mother modeling positive behaviors

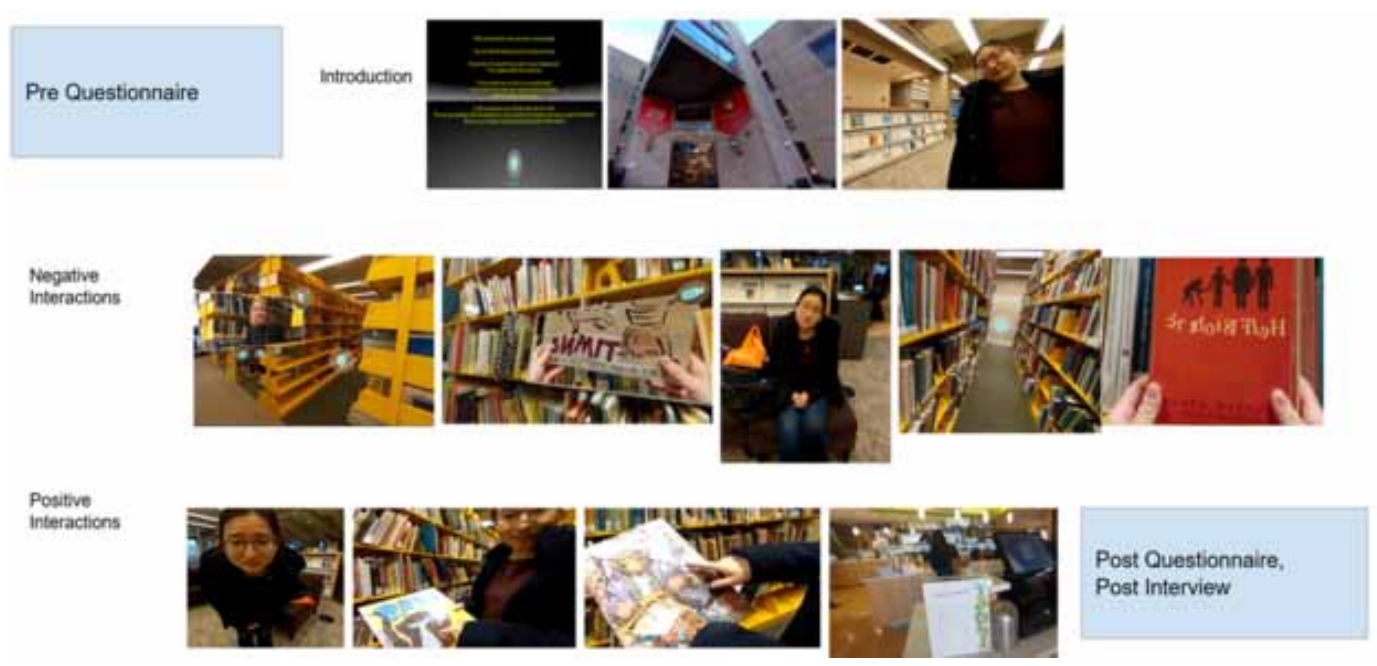

child, who volunteers to find the book Monkey Business. Then, the participant can choose to move to the bookstore bookstacks, and search for the correct book through an interactive segment where they can select several books from shelves. Unfortunately, all the words in the titles are jumbled and not understandable. They then return to the mother unsuccessful, and the mother expresses disappointment. The child insists that they can find the book, so goes back into the bookstacks. In the next interactive segment, the search continues as the participant selects books and flips through them, but cannot understand any of the words. The child's perspective is increasingly distressed, indicated by sounds of heavy breathing, heartbeats, and sobbing. After being unsuccessful again, the crying child returns to the mother. The mother then changes her approach and, instead of being disappointed, goes with the child into the bookstacks and models appropriate behaviors to help reading (e.g., holding a book and reading each syllable of the text). After a few attempts, they find the book.

Finally, the narrator reflects on the fact that the experience was just one of many such in their childhood, and reveals that, even as an adult, they have issues reading in common contexts such as ordering food from a restaurant menu or reading a contract. These final memories are shown through short non-interactive segments.

\section{Design and Metrics}

The current study is intended as a pilot to determine if such a VR experience can cause changes in participant empathy and what design factors are important in constructing the experience. Our quasiexperimental approach follows a pretest-posttest design where all participants experienced the VR experience, and measures were collected before and after the activity. Pre- and post-study survey questions measured participant's change in empathy, anxiety, and perspective-taking. Pre-study survey questions also collected information about participant demographics, and post-study survey and interview collected information about their reaction to the VR activity, including emotional engagement and immersion.

We employed quantitative and qualitative methods to explore the feasibility of this VR experience to change participant empathy, and to understand what factors contribute to the effectiveness of the experience. Differences in pre-post changes in scores were calculated using Wilcoxon paired samples test; post-test measures were checked against neutrality using one-sample Wilcoxon signed rank test; and correlations between variables were typically calculated using Spearman correlations. Due to the 
exploratory nature of this study we report tests that reached significant of $\mathrm{p}<0.1$ as well as $\mathrm{p}<0.05$, and emphasize discussion of qualitative data. For qualitative analysis, one researcher performed thematic coding to extract the themes discussed below.

The following metrics were collected during the study. Survey and interview questions are listed in Appendix A and B.

\section{Demographics, Caretaking Type and Previous VR Experience (Pre-Questionnaire)}

The pre-questionnaire included traditional demographic questions about age, gender identity, ethnic identity, and level of education. It also included caregiving related questions, which covered years caring for children, type of care provided (parent, teacher, or other), ages of children cared for, and whether any of the children cared for had any kind of disability. The survey also asked participants about any previous experience in VR. These questions were created by the researchers.

\section{Empathy, Perspective-Taking and Anxiety (Pre- and Post-Questionnaire)}

To measure empathy this study used an adapted version of the Interpersonal Reactivity Index - IRI (Davis, 1980). In addition to the IRI questions, metrics included custom questions on anxiety, parent perspective-taking, child perspective-taking, and two items from the Adult and Adolescent Parenting Inventory (AAPI) that measures parenting skills. The IRI questionnaire measuring empathy covered the following dimensions:

- Fantasy scale: Captures tendency to put one's self in the shoes of a fictitious character.

- Empathic concern: Evaluates strength of feelings of sympathy and concern towards unfortunate others.

- Perspective taking: Adaptability to psychological point of view of others.

- Personal distress: "Measures self-oriented feelings of personal anxiety and unease in tense interpersonal settings."

\section{Behavioral Questions - Donation and Interest (Pre and Post Questionnaire)}

The study included questions to gauge participants' affinity to caring about children who have reading difficulties. The questions were created by the researchers, and the following question types were asked in both pre and post questionnaires:

- Donating to a charity focused on dyslexia: Participants were asked if they were willing to donate to a charity focused on addressing dyslexia, if the chance arose in the following two weeks.

- Lab research direction: Participants were asked to select one of four possible research areas for our lab to focus on in the future.

- Searching for information about dyslexia: Participants were asked about their intent to search for information about dyslexia in the following two weeks.

\section{Presence During VR Experience (Post-Questionnaire)}

The post questionnaire also measured the participants' feeling of presence and immersion in during the experience, by using a version of the Presence Questionnaire (Witmer, Jerome and Singer, 2005). This questionnaire measures participants' subjective experiences in the immersive experience such as perceived realism, perceived ability to act, possibility to examine, interface quality.

\section{Interview About Emotional Reactions and Design Features (Post Interview)}

Following completion of the post-survey, participants were asked open ended questions (created for this research) to more deeply reveal the factors that influenced their emotional reactions, as well as to provide feedback on the design of the experience. 


\section{Participants}

Convenience sampling was used to recruit different types of caregivers, defined as any person with significant experience interacting with children, including present and previous educators, parents, and other caregivers such as grandparents, babysitters, etc. Participants were recruited from advertisements at the [anonymized university's teacher education] program, and at [anonymized city] schools and childcare centers. 27 participants were recruited for this study (16 female, 10 male, 1 non-binary gender). 4 participants self-identified as being "parent and teacher"; 16 identified as "parent"; 5 identified as "teacher"; and 2 as "other" (specifically, an aunt and a babysitter).

\section{Protocol}

At the start of the study activity, researchers introduced the activity to participants and acquired written consent. The participant was then asked to complete a pre-survey on a laptop computer lasting roughly 10 minutes. Afterwards, the researcher demonstrated how to put on the VR Headset (an Oculus Go) and explained using the controller to navigate the virtual world, as well as using a rotating chair (on which the participant is seated) to explore the 360 degree environment. The researcher then initiated the experience, asking the participant to put on the headset and begin when ready. The experience lasted roughly 15 minutes. After the experience was over, the participant was asked to take a postsurvey on the computer, followed by an interview with the researcher, lasting roughly 20 minutes.

\section{RESULTS}

\section{RQ1: What Kind of Emotional Reaction Does This Experience Create in Participants? How Strong is the Emotional Reaction?}

\section{Emotional Reaction and Relatability}

Post-survey questions measured the strength of emotional and empathetic reaction to the experience, where answer options ranged from No Reaction to Strong Reaction. After the experience, participants reported significantly higher-than-neutral emotional reaction and empathetic reaction (Figure 3) $(\mathrm{V}>120, \mathrm{p}<0.01)$. There was a significant positive correlation between emotional reaction and the pre-post change in willingness to donate $(r=0.56 ; \mathrm{p}<0.05)$.

Figure 3. Participant reactions to the question "How empathetic did you feel toward the child's struggles?" (red) and "Please rate your emotional reaction to the experience" (blue). All answers were significantly higher than neutral (1=no reaction, $5=$ strong reaction).

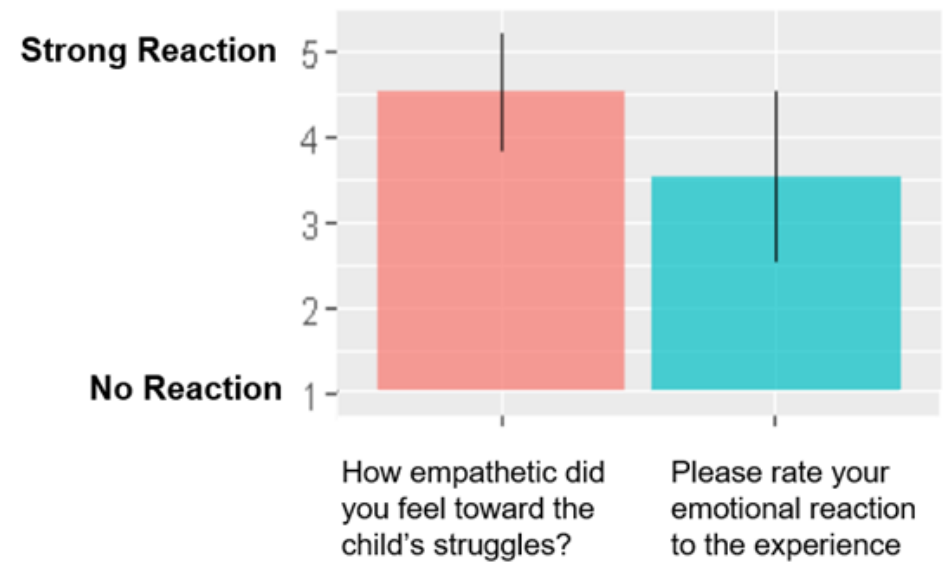


Participants were asked during interviews whether they related to the child and parent in the experience (Figure 4). 80\% of participants responded that they found the child relatable. Answering that the child was relatable was positively correlated to increase in willingness to make a donation towards helping children with reading difficulties $(r=0.73 ; \mathrm{p}<0.05)$. When asked if the parent was relatable, $65 \%$ of participants indicated yes, but note this answer is strongly influenced by whether the participant was a parent or teacher. In a similar but weaker relationship, the participants who found the parent relatable were also more likely to display an increased willingness to donate $(\mathrm{r}=0.56 ; \mathrm{P}<0.1)$.

On average, participants who were teachers (regardless of parenting experience) reported less connection to the parent of the story and reported on average a milder emotional reaction than participants who were parents but were not teachers. One possible explanation for this is that teachers may be less sensitive to emotional influence due to the professional training they receive and/or their continued exposure to a broad range of student profiles. Further, another factor may be participants' previous exposure to reading difficulties: all teacher-only participants responded that they had at some point dealt with children with reading difficulties or dyslexia on the pre-survey. this was a diametrical opposite to the responses of parent-only participants, where none had exposure to dealing with children with reading difficulties or dyslexia.

\section{Types of Emotional Reactions}

During interviews, participants reported different kinds of reactions touching on various emotions, illustrated through the quotes below. Some participants experienced anxiety ("When I had to go back and try again with the heartbeat, it was poignant because I think my expectation was that I was going to find the book, but I couldn't. I felt mostly the anxiety that a child could feel."). Others felt sorrow ("This was not a very good situation. The child was not understood and I couldn't see why he was not understood. He was hurt, then he was asked to forgive. And of course in this situation the child

Figure 4. Relatability of the child (top) and parent (bottom) according to the type of participant: Parent only, Parent Teacher, Teacher only. Numbers indicate the number of participants. "No answer" was assigned if the participant answered, but provided no clear indication of agreement / disagreement to the statement.

\section{Was the Child relatable?}

Parent Only

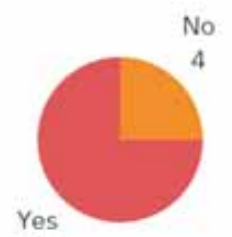

12

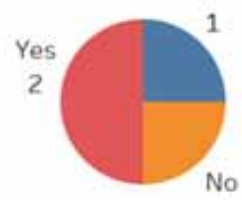

1
Teacher Only
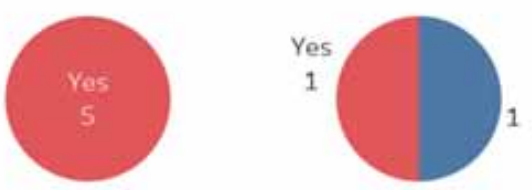

Was the Parent relatable?
Teacher Parent

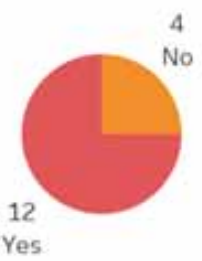

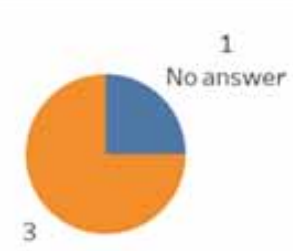

No
Teacher Only

Other Caretaker Type
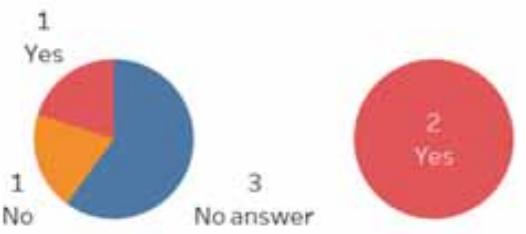
would forgive because he wants things to be over and longs for a hug and wants to go back to his mother's warmth or whatever, but I didn't forgive the mother."). Still others experienced frustration ("Felt frustrated for that child...that they would be very difficult to achieve what the family member wanted, with the set of directions that they were given. So maybe I feel frustrated, empathetic and sad."). Some experienced hopelessness ("Almost hopeless. I wanted to accomplish this goal but didn't have the skills to do it."). Some participants related to the parent ("I felt sad or the child, but sympathetic for the mom.") or related to the child ("I could feel what the child felt. I could imagine being in his shoes. I could imagine how my daughter would feel if she was in this situation.").

\section{RQ2: To What Degree Does This Experience Change Participant Attitudes or Behaviors Towards Struggling Children?}

We evaluated changes in participant empathy scores on the Interpersonal Reactivity Index questionnaire, administered before and after the study. Figure 5 shows the change in scores.

Significant pre-post differences were detected in the dimension of Perspective Taking $(Z=2.9$, $\mathrm{p}<0.01$ ), where participants had significantly lower post-test scores. This category contained questions such as "Before criticizing somebody, I try to imagine how I would feel if I were in their place". One potential explanation is that participants discovered during the experience their perspective taking ability was not as high as they thought (i.e., realized that child's experience is unusual and not as expected); thus, their post-experience self-reported scores on perspective taking would be lowered to correct for this.

On average, participants responding that the experience will change how they interact with children was correlated with a drop in Perspective Taking empathy scores $(r=-0.43 ; \mathrm{p}<0.05)$. Similarly, participants who demonstrated a lessened ability for perspective taking on the post- compared with the pre-survey also demonstrated an increased willingness to search for information on dyslexia within 2 weeks after completing the experience on the post-survey compared to when asked that same question on the pre-survey $(\mathrm{r}=-0.68, \mathrm{p}<0.05)$. The fact that participant scores dropped might indicate that this experience caused participants to realize that one's ability to take the perspective of others is not actually as high as previously believed, and for such participants this prompted an increased willingness to take action.

Post questions also asked participants questions about their subjective attitude towards children. The results indicate that the majority of participants felt the experience impacted their perception of children, indicated by questions such as: "Do you feel this experience will make you think more about how you respond to your own children?" (73\% yes) "Do you feel this experience will make you behave differently towards your own children or students?" (57\% yes) "Do you feel this experience changed you in any way?" (52\% yes)

\section{RQ3: What Factors of the Experience Influence Participant Emotions, Attitude/Behavior Changes?}

Although these results are unclear in showing possible long-lasting effects, correlational analysis shows that long-lasting effects might occur in some participants more than others (Figure 6). The increase in participant willingness to make a donation was stronger in those participants who found

Figure 5. Changes in empathy subdimension scores (Range 0-4. Negative values mean the post-experience score was lower than the pre-experience score.)

\section{Change in self-reported score (post-pre)}

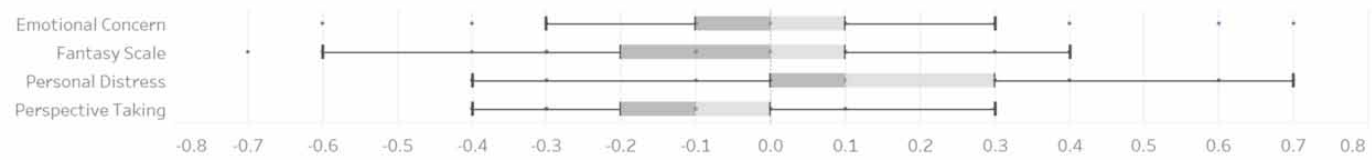


Figure 6. Significant correlations between the study variables

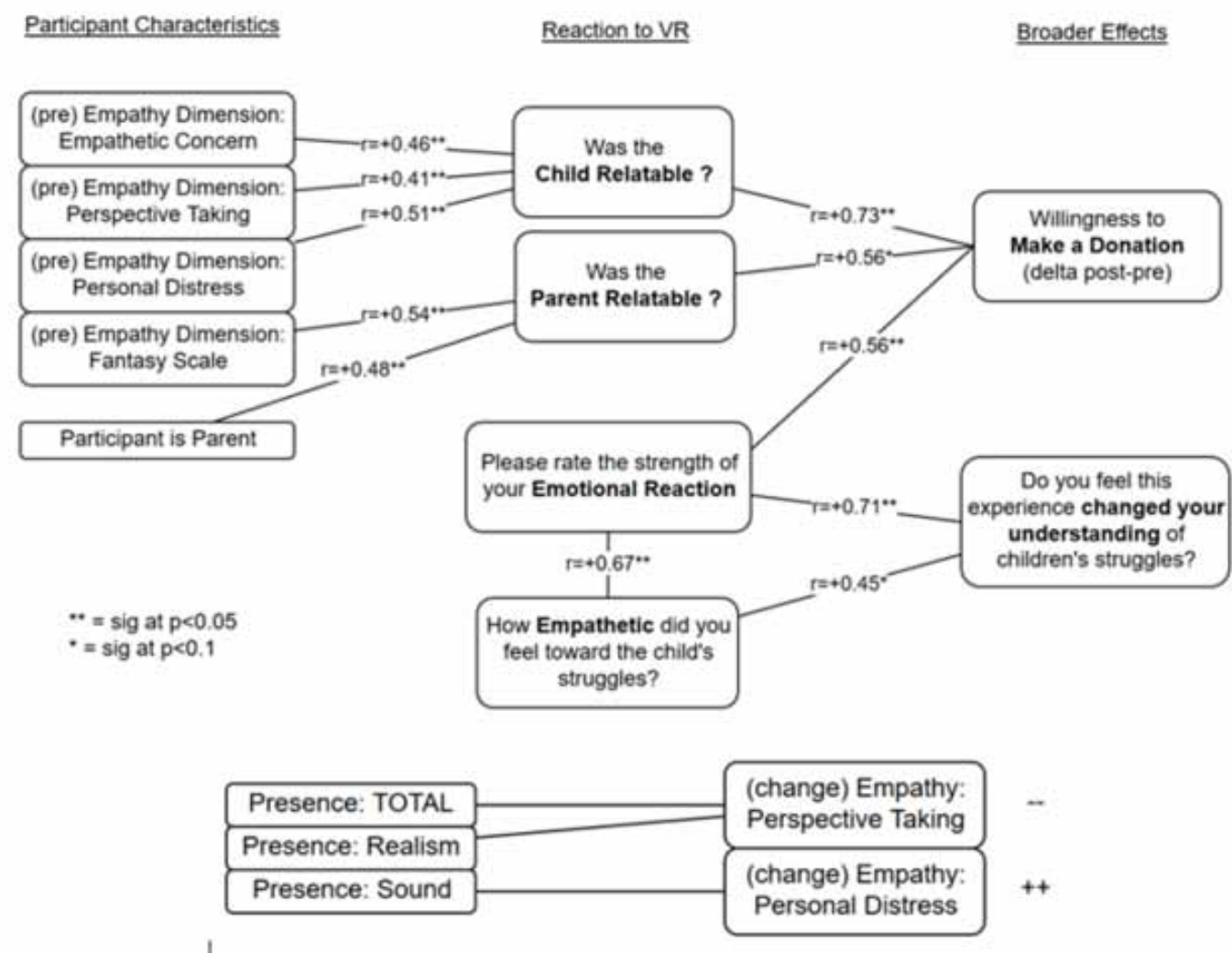

the child relatable, found the parent relatable, and had a stronger emotional reaction to the experience. Further, participants' self-report of possible changes in understanding of children's struggles was stronger when they had a stronger emotional reaction to the experience, or a stronger feeling of empathy towards the child.

Feelings of empathy for the child was significantly correlated to strength of emotional reaction measured in the post survey $(\mathrm{r}=0.67 ; \mathrm{p}<0.05)$. Participants who reported a higher emotional reaction to the experience on the post-survey were also more likely to want to donate to a charity addressing dyslexia on the post-survey compared with the pre-survey $(\mathrm{r}=0.56 ; \mathrm{p}<0.05)$, and participants responding that the child was relatable in the interview showed a significant association to a higher willingness to donate $(\mathrm{r}=0.73 ; \mathrm{p}<0.05)$. Participants who found the parent relatable on the post-survey also showed a similar willingness $(\mathrm{r}=0.65 ; \mathrm{p}<0.05)$. Interestingly, participants who were both parents and teachers at the same time expressed a lesser willingness to donate in the post-survey compared with the pre-survey $(\mathrm{r}=-0.58 ; \mathrm{p}<0.05)$.

It is also important to note that teacher-parents tended to find the parent figure in the VR experience less relatable $(\mathrm{r}=-0.52 ; \mathrm{p}<0.05)$, which might indicate that character relatability is at the core of the change in willingness to donate. This potential interpretation should be taken with caution, however, since only 4 teacher-parents were in the sample, and since being a teacher only was not found to have a significant association to parent relatability - a finding that, if validated, could help to explain why teacher-parents have, on average, not found the parent figure to be relatable. 


\section{Mechanism for Relatability}

When asked if the child was relatable, participants who responded positively frequently reported that they remembered similar experiences from their own childhood. Additionally, participants who had high scores on empathy before taking the study were correlated to having a strong feeling of relatability towards the child. This hints that the power of immersive media experiences to elicit emotional reactions is related to participants' ability to project themselves into the experience. Conversely, participants who did not feel connected to the child's experience reported lacking similar childhood experiences. In response to whether they felt like the child was relatable, participants mentioned projecting themselves into the role of the child ("the child hasn't said much... it was a fairly open experience where I could project myself on ... open ended for me to assume what the child is saying.") and frequently connected to experiences from their own childhood ("I could understand what the child was feeling. The child wanted to help. When I was a kid I also wanted to help and didn't want to let my parents down and it felt frustrating when I couldn't help."; or "I can see that children want to do things on their own and be confident in that. They get confused and try to accomplish the task. In my childhood I struggled with things, I didn't have dyslexia but I had reading difficulties. I felt I didn't ask for help when I needed it."), and some participants explicitly reported not feeling relatable to the child because of a lack of similar experiences ("[The child was not relatable] not to me personally. Because I didn't experience a situation like this growing up”)

\section{Teachers vs. Parents}

We observed differences between participants who were teachers vs. those who were not. Teachers reported on average a milder emotional reaction than parents. Teachers scored lower when asked to rate their emotional reactions, and whether the experience changed their understandings of children's struggles (Figure 7). Additionally, participants who were not teachers showed a higher increase in donation willingness after completing the experience compared to before completing it (Figure 8). On average, as participant years of experience caring for children increased, they were less likely to donate in the post survey $(\mathrm{r}=-0.65 ; \mathrm{p}<0.05)$.

Figure 7. Post-experience survey question differences between participants who had teaching experience vs. those that did not (Range 1-5)

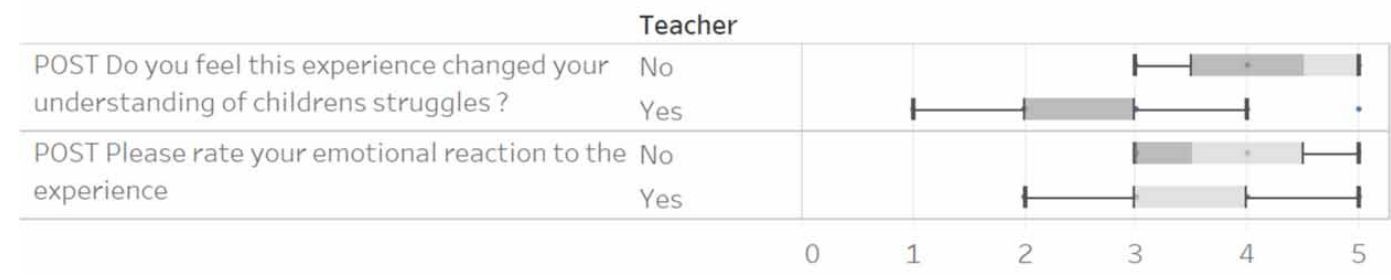

Figure 8. Difference in post-pre survey scores on willingness to make a donation between participant agreement with the statements, for participants who had teaching experience vs. those that did not (Range $0-4$ )

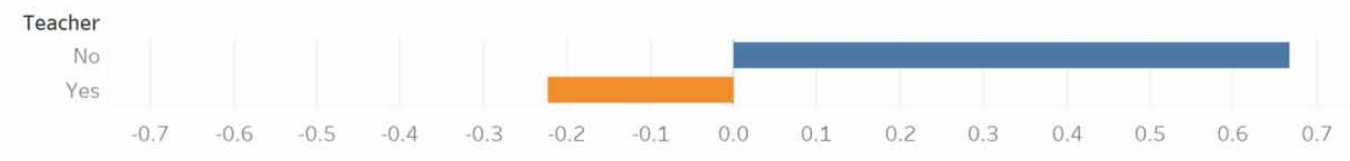


Parents and teachers in our sample had varying answers to post-interview questions. Participants who were teachers were less likely to respond "yes" when asked if the experience changed them in any way, and whether it will change how they behave towards children they care for. Participants who were parents related more to the parent figure in our VR experience $(r=0.40 ; p<0.05)$, while parents who were teachers did not relate to the parent figure $(\mathrm{r}=-0.43 ; \mathrm{p}<0.05)$. This negative correlation for teacher-parents also surfaced when asked during the interview about relating to the parent figure $(\mathrm{r}=-0.52 ; \mathrm{p}<0.05)$. Teachers also tended to find the parent in the VR experience to not be relatable, although both teachers and non-teachers found the child relatable. (Figure 9)

One explanation for this observation is that teachers may be less sensitive to emotional influence in pedagogical settings due to the professional training they receive and/or their continued exposure to a broad range of student profiles. This explanation is plausible in our study, given that all teacheronly participants responded that they had at some point dealt with children with reading difficulties or dyslexia on the pre-survey. This was in contrast to the responses of parent-only participants; where none had exposure to dealing with children with reading difficulties or dyslexia. Teachers who reported that the experience will not change how they behave with their children mentioned they are already familiar with the topic (e.g. "I don't know if it changed me but it was a good reminder.", or "I had a lot of practice with empathy already.”)

\section{RQ4a: What Design Features Enhanced the Participants' Experience?}

Participant answers identified several features that enhanced the experience:

- Heartbeat: Eight participants who mentioned the heartbeat in the interview said it made the experience feel more vivid, mentioning things such as "My heart rate increased when I heard the increased heart rate." and "I had to talk myself into being calm after hearing the heartbeat".

- Child crying: 3 participants commented on how the sound effects of the child crying made them feel more anxious and drawn into the child's situation, such as "[It] felt little emotional when she started crying" and "At some points she started crying because she couldn't find the book and I thought that was relatable."

- Realistic narrative: some participants commented on how this scenario could unfold in a real-life situation: children try to please their parents and get upset when they cannot, through statements such as "Yes. I would act in a similar manner to her in that situation." and "I felt like the same hustle for parents everyday, and that this could happen in real life".

Figure 9. Post interview differences between participant agreement with the statements, for participants who had teaching experience vs. those that did not. (Range 0-1)

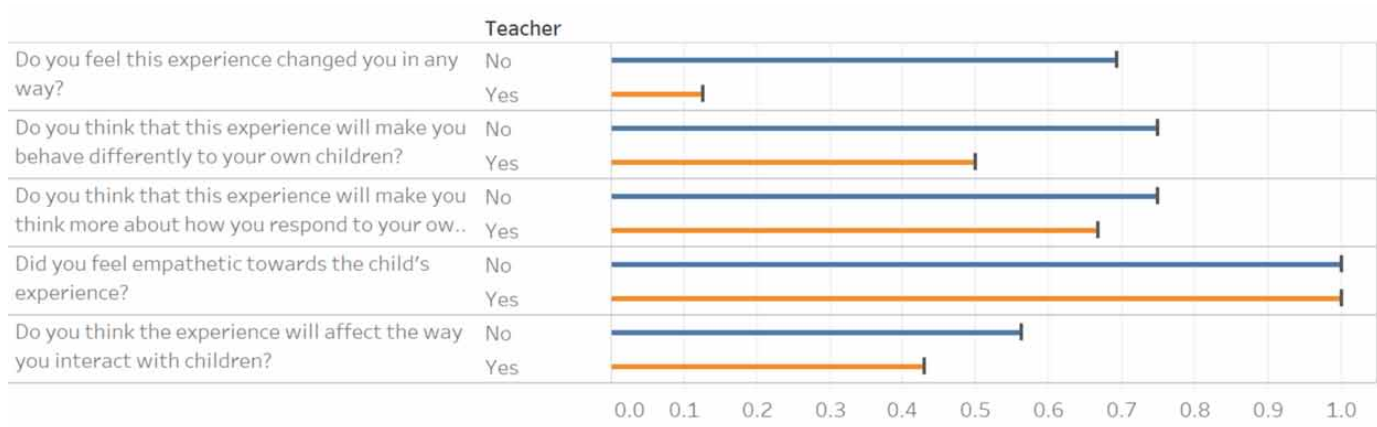




\section{RQ4b: What Design Features Hindered the Participants' Experience?}

Participant answers identified several features that could be improved about the experience:

- Volume of books: Seven participants thought the amount of books to select in the experience (6 books) was too much. However, one participant thought more books would intensify the experience: "It could be made more overwhelming, more books, more pressure, because for children, the real situation would be much more overwhelming than what we see in the experience."

- Mother character: Participants who found the parent figure relatable also had a higher empathic concern score (as reported earlier), indicating that parent relatability is an important design aspect. It would be beneficial to invest in a professional actor, since many participants explicitly stated that the mother figure (a graduate student) did not feel genuine; reasons ranged from "she looks too young" and "doesn't resemble my mom" to "she didn't seem to be in a hurry or stressed enough".

- Layout of the space: At least 5 participants pointed out that the experience did not take place at the children's book section of a bookstore, making the experience seem staged and inauthentic, mentioning things such as "It felt like they [the books] were not laid out like the children's library would be." and "Was odd to be at [our school], not a real bookstore."

- Duration: Some participants questioned if the effect of this experience will be a lasting one, given that it is just 15 minutes.

\section{DISCUSSION}

The immersive experience in this study, while short in duration and experimental in nature, generated strong emotions in a majority of the participants. These emotions included sadness, anxiety and frustration. Further, all participants reported significantly higher-than-neutral emotional reactions to the experience. The outcomes of this pilot study may aid in enhancing emotions related to empathy, both by improved design based on heuristics from the findings (e.g., evoke early childhood experiences participants are likely to have experienced) and through customizing the experience to each participant's culture, background and role (e.g., develop different experiences for parents and for teachers who are not parents). Also, increasing the relatability of the parent and child depicted in the experience by personalizing these to each participant could enhance the impact of the experience(e.g., the simulated parent's gender and ethnicity could be customized based on information in the participant pre-survey). Below we reflect on some of the factors and effects observed in this exploratory study, making suggestions for design heuristics.

\section{Design Strategies to Increase Effectiveness}

\section{Predispositions}

The impact of the VR experience is influenced by participants' predispositions to empathy and perspective-taking. Participants' predispositions towards empathetic behaviors influenced their ability to relate to the parent/child depicted in the VR experience, which in turn influenced their willingness to change behaviors through making a donation. Overall, people who found the characters more relatable and people who came into the study with higher empathic concern, perspective taking and personal distress scores were more likely to report attitude change and display in the form of an increased willingness to donate to a cause relating to children with reading disabilities or dyslexia. This suggests that VR empathy experiences can be designed to adapt to the user's predispositions, for example being informed by information collected through pre-questionnaires or physiological data, and adapt the intensity of content to the user. 


\section{Projection}

The impact of the VR experience is influenced by participants' ability to project themselves in the experience, which is mediated by background experiences as children, or experiences being in other relatable situations (e.g., parenting, teaching). This suggests that designers of VR empathy experiences should build on popular childhood activities relatable to most people, or on personalized childhood activities based on information collected from interviews with the target audience.

\section{Narrative With Sensory Enhancements}

The realistic narrative (situation) enhanced the relatability of the characters. Also, scores on immersive presence, which can be enhanced by narrative, social, and sensory factors, correlated to participant impact. For example, feelings of anxiety and sadness were enhanced by auditory factors such as increased heart rate and the child crying. Overall, creating a relatable character in a relatable situation changed some people's behaviors. Specifically, relatability to the child or to the parent were both correlated with willingness to make a donation. This suggests that VR experiences can potentially be customized based on information in the pre-interview, for example adapting the gender and ethnic/ cultural background of the virtual child and environment, or adjusting the virtual heartbeat and ambient stressors based on real-time information about the user's actions in the virtual experience.

\section{Teachers in Contrast to Parents}

Teachers reported lower emotional reactions and lower relatability to the parent than parents did, as well as lower ratings on whether the experience changed their understandings of children's struggles. While parents generally related to the parent figure, teachers who were parents did not find the parent relatable. This is possibly due to professional exposure to children's emotional reactions, as well as higher exposure to children with reading difficulties. Teachers had higher exposure to children with dyslexia than parents and, as participant years of experience caring for children increased, they were less likely they were to donate in the post survey. In contrast, participants who were not teachers showed a higher increase in donation willingness after completing the experience compared to before completing it. This can be potentially influenced by design (for example, by creating more emotionally charged situations for teachers than for parents), and future work should investigate if teachers empathy should be stimulated in different ways than parents.

\section{Implications for Potential Impact}

\section{Behavioral Change}

This study suggests that designing VR experiences evoking relatability and emotional reactions have the potential to change participant behaviors. Higher emotional reaction was correlated with higher change in willingness to make a donation. However, the pilot study was not designed to elucidate the complex factors that link emotional responses to behavioral change, or to measure long term behavior change, and these are issues on which more research is needed.

\section{Feeling Empathy vs. Changing Empathy}

This pilot study is not designed as an intervention to increase long term empathy in users. Instead, emotional factors potentially related to sustained changes in empathy were studied. Building capacity in empathy towards children struggling with literacy would likely require a series of different types of experiences with a higher dosage over a period of time. In the design of capacity building experiences, this study suggests the following two issues should be addressed: First, on average, participant evaluation of their own personal distress increased after completing the experience. Distress in itself does not necessarily induce empathy. Second, on average, participants' perspective taking score fell. This might indicate that participants discovered they were moved by the VR experience more than they anticipated, yet realized they were not as good in taking the perspective of others as they 
previously thought. More research is needed to determine the cause of this outcome, especially since increasing perspective taking is likely related to increasing empathy.

Overall, this study suggests that using video-based virtual reality experiences is a promising approach to building adult capacity to help children's early literacy though inducing attitudinal and behavior shifts. Such a strategy is becoming increasingly affordable and scalable as the requisite technology infrastructure spreads through society.

\section{LIMITATIONS AND SUGGESTIONS FOR FUTURE RESEARCH}

The findings from this pilot study are suggestive rather than conclusive or generalizable. The small convenience sample in this pilot study may not be representative of the full population of adults who have caregiver roles related to early literacy, and contributes to low statistical power; additionally, the multiple correlational tests pose an increased risk of Type I statistical errors. In addition, the dosage of the treatment is low, which is likely to produce only transient effects on attitudes and behavior rather than sustained shifts. A more comprehensive study with multiple self-reports and behavioral measures would be necessary to have confidence in the metrics. Future work could pursue a true experimental design, involving a baseline control group which is exposed to the same narrative experience in a non-immersive medium, for instance comparing browser-based experiences vs. headset-based VR. Contrasting experiences that are primarily passive vs those in which participants have substantial agency and choice is another important consideration.

\section{ACKNOWLEDGMENT}

We wish to thank the dedicated research assistants who aided in this research: Merry Chin, Tara Nair, and Mitch Scuzzarella. The Reach Every Reader project at Harvard University provided the funding for this effort. That project is supported by the Chan-Zuckerberg Initiative. The discussions and conclusions about this project are the authors and do not reflect the views of the sponsors. We also thank the study participants, and Amanda Taylor and the Harvard Teacher Education Program for their assistance with participant recruitment.

\section{FUNDING AGENCY}

Open Access Funding for this article has been provided by IGI Global. 


\section{REFERENCES}

Accardo, A., \& Xin, J. (2017). Using Technology-Based Simulations to Promote Teacher Candidate Parental Collaboration and Reflective Instructional Decision Making. Journal of Technology and Teacher Education, 25(4), 475-494. Retrieved February 17, 2020, from http://www.learntechlib.org/p/173335/

Adefila, A., Graham, S., Clouder, L., Bluteau, P., \& Ball, S. (2016). myShoes-The future of experiential dementia training. The Journal of Mental Health Training, Education and Practice, 11(2), 91-101. doi:10.1108/ JMHTEP-10-2015-0048

Archer, D., \& Finger, K. (2018). Walking in Another's Virtual Shoes: Do 360-Degree Video News Stories Generate Empathy in Viewers? Academic Press.

Bachen, C. M., Hernández-Ramos, P. F., \& Raphael, C. (2012). Simulating REAL LIVES: Promoting global empathy and interest in learning through simulation games. Simulation \& Gaming, 43(4), 437-460. doi: $10.1177 / 1046878111432108$

Badiee, F., \& Kaufman, D. (2014). Effectiveness of an Online Simulation for Teacher Education. Journal of Technology and Teacher Education, 22(2), 167-186.

Bailenson, J. (2018). Experience on demand: What virtual reality is, how it works, and what it can do. Norton.

Bailey, J., \& Bailenson, J. (2017). Considering virtual reality in children's lives. Journal of Children and Media, 11(1), 107-113. doi:10.1080/17482798.2016.1268779

Banakou, D., Groten, R., \& Slater, M. (2013). Illusory ownership of a virtual child body causes overestimation of object sizes and implicit attitude changes. Proceedings of the National Academy of Sciences of the United States of America, 110(31), 12846-12851. doi:10.1073/pnas.1306779110 PMID:23858436

Banakou, D., Hanumanthu, P. D., \& Slater, M. (2016). Virtual embodiment of white people in a black virtual body leads to a sustained reduction in their implicit racial bias. Frontiers in Human Neuroscience, $10,601$. doi:10.3389/fnhum.2016.00601 PMID:27965555

Bondie, R., Macenido, Z., \& Dede, C. (in press). Interaction principles for digital puppeteering to promote teacher learning. Journal of Research in Teacher Education.

Brook, C., Pedlar, M., Abbott, C., \& Burgoyne, J. (2016). On stopping those things that are not getting us to where we want to be: Unlearning, wicked problems and critical action learning. Human Relations, 69(2), 369-389. doi: $10.1177 / 0018726715586243$

Christensen, R., Knezek, G., Tyler-Wood, T., \& Gibson, D. (2011). simSchool: An online dynamic simulator for enhancing teacher preparation. International Journal of Learning Technology, 6(2), 201-220. doi:10.1504/ IJLT.2011.042649

Cohen, J., Wong, V., Krishnamachari, A., \& Berlin, R. (2020). Teacher coaching in a simulated environment. Educational Evaluation and Policy Analysis, 42(2), 208-231. doi:10.3102/0162373720906217

Dalinger, T., Thomas, K. B., Stansberry, S., \& Xiu, Y. (2020). A mixed reality simulation offers strategic practice for pre-service teachers. Computers \& Education, 144, 103696. doi:10.1016/j.compedu.2019.103696

Davis, M. H. (1980). A multidimensional approach to individual differences in empathy. Catalog of Selected Documents in Psychology, 10, 85.

Dyer, E., Swartzlander, B., \& Gugliucci, M. (2018). Using virtual reality in medical education to teach empathy. Journal of the Medical Library Association: JMLA, 106(4), 498-500. doi:10.5195/JMLA.2018.518 PMID:30271295

Entwisle, D. R., Alexander, K. L., \& Olson, L. S. (1997). Children, schools and inequality. Westview Press.

Farrant, B. M., \& Zubrick, S. R. (2012). Early vocabulary development: The importance of joint attention and parent-child book reading. First Language, 32(3), 343-364. doi:10.1177/0142723711422626

Fonagy, P., Gergely, G., \& Target, M. (2007). The parent-infant dyad and the construction of the subjective self. Journal of Child Psychology and Psychiatry, and Allied Disciplines, 48(3-4), 288-328. doi:10.1111/j.14697610.2007.01727.x PMID:17355400 
Formosa, N. J., Morrison, B. W., Hill, G., \& Stone, D. (2018). Testing the efficacy of a virtual reality-based simulation in enhancing users' knowledge, attitudes, and empathy relating to psychosis. Australian Journal of Psychology, 70(1), 57-65. doi:10.1111/ajpy.12167

Gondoli, D. M., \& Silverberg, S. B. (1997). Maternal emotional distress and diminished responsiveness: The mediating role of parenting efficacy and parental perspective taking. Developmental Psychology, 33(5), 861-868. doi:10.1037/0012-1649.33.5.861 PMID:9300219

Greitemeyer, T., Osswald, S., \& Brauer, M. (2010). Playing prosocial video games increases empathy and decreases schadenfreude. Emotion (Washington, D.C.), 10(6), 796-802. doi:10.1037/a0020194 PMID:21171755

Gundel, E., Piro, J. S., Straub, C., \& Smith, K. (2019). Self-Efficacy in Mixed Reality Simulations: Implications for Preservice Teacher Education. Teacher Educator, 54(3), 244-269. doi:10.1080/08878730.2019.1591560

Hamilton-Giachritsis, C., Banakou, D., Quiroga, M. G., Giachritsis, C., \& Slater, M. (2018). Reducing risk and improving maternal perspective-taking and empathy using virtual embodiment. Scientific Reports, 8(1), 2975. doi:10.1038/s41598-018-21036-2 PMID:29445183

Hirsh-Pasek, K., Adamson, L. B., Bakeman, R., Owen, M. T., Golinkoff, R. M., Pace, A., Yust, P. K. S., \& Suma, K. (2015). The contributions of early communication quality to low-income children's language success. Psychological Science, 26(7), 1-13. doi:10.1177/0956797615581493 PMID:26048887

Hoff, E. (2003). The specificity of environmental influence: Socioeconomic status affects early vocabulary development via maternal speech. Child Development, 74(5), 1368-1378. doi:10.1111/1467-8624.00612 PMID:14552403

Ip, H. H. S., Wong, S. W. L., Chan, D. F. Y., Byrne, J., Li, C., Yuan, V. S. N., Lau, K. S. Y., \& Wong, J. Y. W. (2018). Enhance emotional and social adaptation skills for children with autism spectrum disorder: A virtual reality enabled approach. Computers \& Education, 117, 1-15. doi:10.1016/j.compedu.2017.09.010

Jütten, L., Mark, R., Maria Janssen, B., Rietsema, J., Dröes, R., \& Sitskoorn, M. (2017). Testing the effectivity of the mixed virtual reality training Into D'mentia for informal caregivers of people with dementia: Protocol for a longitudinal, quasi-experimental study. BMJ Open, 7(8), E015702. doi:10.1136/bmjopen-2016-015702 PMID:28827242

Katz, Y. (1999). Kindergarten Teacher Training Through Virtual Reality: Three-Dimensional Simulation Methodology. Educational Media International, 36(2), 151-156. doi:10.1080/0952398990360211

Kohn, A. (1998). Raising children who care. NAMTA Journal, 25, 185-206.

Lorenzo, G., Lledó, A., Pomares, J., \& Roig, R. (2016). Design and application of an immersive virtual reality system to enhance emotional skills for children with autism spectrum disorders. Computers \& Education, 98, 192-205. doi:10.1016/j.compedu.2016.03.018

Norman, P. C. (2016). Teacher attitudes and perceptions of low and high socioeconomic status students. All Graduate Theses and Dissertations. 4873. https://digitalcommons.usu.edu/etd/4873

Peck, T. C., Seinfeld, S., Aglioti, S. M., \& Slater, M. (2013). Putting yourself in the skin of a black avatar reduces implicit racial bias. Consciousness and Cognition, 22(3), 779-787. doi:10.1016/j.concog.2013.04.016 PMID:23727712

Pellegrini, A., \& Galda, L. (2003). What gets learned in joint reading?'. On reading books to children: Parents and teachers. Lawrence Erlbaum.

Psychogiou, L., Daley, D., Thompson, M. J., \& Sonuga-Barke, E. J. (2008). Parenting empathy: Associations with dimensions of parent and child psychopathology. British Journal of Developmental Psychology, 26(2), 221-232. doi:10.1348/02615100X238582

Rosenberg, R., Baughman, S., \& Bailenson, J. (2013). Virtual superheroes: Using superpowers in virtual reality to encourage prosocial behavior. PLoS One, 8(1), E55003. doi:10.1371/journal.pone.0055003 PMID:23383029

Rowe, M. L., Leech, K. A., \& Cabrera, N. (2017). Going beyond input quantity: Wh-questions matter for toddlers' language and cognitive development. Cognitive Science, 41, 162-179. doi:10.1111/cogs.12349 PMID:26923546 
Schutte, N. S., \& Stilinović, E. J. (2017). Facilitating empathy through virtual reality. Motivation and Emotion, 41(6), 708-712. doi:10.1007/s11031-017-9641-7

Seidel, T., Blomberg, G., \& Renkl, A. (2013). Instructional strategies for using video in teacher education. Teaching and Teacher Education, 34, 56-65. doi:10.1016/j.tate.2013.03.004

Seinfeld, S., Zhan, M., Poyo-Solanas, M., Barsuola, G., Vaessen, M., Slater, M., Sanchez-Vives, M. V., \& de Gelder, B. (2020). Being the victim of virtual abuse changes default mode network responses to emotional expressions. Cortex. Advance online publication. doi:10.1016/j.cortex.2020.11.018

Shin, D. (2018). Empathy and embodied experience in virtual environment: To what extent can virtual reality stimulate empathy and embodied experience? Computers in Human Behavior, 78, 64-73. doi:10.1016/j. chb.2017.09.012

Slater, M., \& Sanchez-Vives, M. V. (2016). Enhancing our lives with immersive virtual reality. Frontiers in Robotics and AI, 3, 74. doi:10.3389/frobt.2016.00074

Spencer, S., Drescher, T., Sears, J., Scruggs, A. F., \& Schreffler, J. (2019). Comparing the Efficacy of Virtual Simulation to Traditional Classroom Role-Play. Journal of Educational Computing Research, 57(7), 1772-1785. doi: $10.1177 / 0735633119855613$

Straub, C., Dieker, L., Hynes, M., \& Hughes, C. (2014). Using virtual rehearsal in TLE TeachLivE ${ }^{\mathrm{TM}}$ mixed reality classroom simulator to determine the effects on the performance of mathematics teachers. 2014 TeachLivE National Research Project: Year 1 Findings. University of Central Florida.

Tropp, L., \& Barlow, F. (2018). Making Advantaged Racial Groups Care About Inequality: Intergroup Contact as a Route to Psychological Investment. Current Directions in Psychological Science, 27(3), 194-199. doi: $10.1177 / 0963721417743282$

Väyrynen, J., Colley, A., \& Häkkilä, J. (2016). Head mounted display design tool for simulating visual disabilities. In Proceedings of the 15th International Conference on Mobile and Ubiquitous Multimedia (pp. 69-73). ACM. doi:10.1145/3012709.3012714

Wijma, E., Veerbeek, M., Prins, M., Pot, A., \& Willemse, B. (2018). A virtual reality intervention to improve the understanding and empathy for people with dementia in informal caregivers: Results of a pilot study. Aging \& Mental Health, 22(9), 1115-1123. doi:10.1080/13607863.2017.1348470 PMID:28691861

Witmer, B. J., Jerome, C. J., \& Singer, M. J. (2005). The factor structure of the Presence Questionnaire. Presence (Cambridge, Mass.), 14(3), 298-312. doi:10.1162/105474605323384654

\section{ENDNOTES}

1 Adapted from https://fetzer.org/sites/default/files/images/stories/pdf/selfmeasures/EMPATHYInterpersonalReactivityIndex.pdf

2 Adapted from https://marketinginvolvement.files.wordpress.com/2013/12/pq-presence-questionnaire.pdf 


\section{APPENDIX A: SURVEY QUESTIONS}

\section{Table 1. Adapted Interpersonal Reactivity Index Questionnaire ${ }^{1}$}

\begin{tabular}{|c|c|}
\hline & Question \\
\hline 1 & I daydream and fantasize, with some regularity, about things that might happen to me. \\
\hline 2 & I often have tender, concerned feelings for people less fortunate than me. \\
\hline 3 & I believe strict discipline is the best way to raise children. \\
\hline 4 & I sometimes find it difficult to see things from the "other person's" point of view. \\
\hline 5 & Sometimes I don't feel very sorry for other people when they are having problems. \\
\hline 6 & I really get involved with the feelings of the characters in a novel. \\
\hline 7 & Before dealing with strangers in general, I feel afraid of an unpleasant situation arising \\
\hline 8 & When children cry, I often think they are misbehaving on purpose. \\
\hline 9 & In emergency situations, I feel apprehensive and ill-at-ease \\
\hline 10 & I am usually objective when I watch a movie or play, and I don't often get completely caught up in it. \\
\hline 11 & I try to look at everybody's side of a disagreement before I make a decision. \\
\hline 12 & When I see someone being taken advantage of, I feel kind of protective towards them. \\
\hline 13 & When a child is struggling, I try to slow down and see the world from their perspective. \\
\hline 14 & I sometimes feel helpless when I am in the middle of a very emotional situation. \\
\hline 15 & I sometimes try to understand my friends better by imagining how things look from their perspective. \\
\hline 16 & I believe parents need to push their children to do better. \\
\hline 17 & Becoming extremely involved in a good book or movie is somewhat rare for me. \\
\hline 18 & I think a child will throw a tantrum just to get their way. \\
\hline 19 & When I see someone get hurt, I tend to remain calm. \\
\hline 20 & Other people's misfortunes do not usually disturb me a great deal. \\
\hline 21 & I feel anxious when I am grocery shopping with my child \\
\hline 22 & I feel that parents should think about their child's point of view. \\
\hline 23 & If I'm sure I'm right about something, I don't waste much time listening to other people's arguments. \\
\hline 24 & After seeing a play or movie, I have felt as though I were one of the characters. \\
\hline 25 & Being in a tense emotional situation scares me. \\
\hline 26 & When I see someone being treated unfairly, I sometimes don't feel very much pity for them. \\
\hline 27 & I am usually pretty effective in dealing with emergencies. \\
\hline 28 & I believe children and adults are similar in how they understand the world. \\
\hline 29 & I am often quite touched by things that I see happen. \\
\hline 30 & I believe that there are two sides to every question and try to look at them both. \\
\hline 31 & I would describe myself as a pretty soft-hearted person. \\
\hline 32 & I feel anxious about going with my child to a bookstore \\
\hline 33 & When I watch a good movie, I can very easily put myself in the place of a leading character. \\
\hline 34 & I tend to lose control during emergencies. \\
\hline
\end{tabular}


Table 1. Continued

\begin{tabular}{|l|l|}
\hline 35 & \multicolumn{1}{|c|}{ Question } \\
\hline 36 & I believe good children always obey their parents. \\
\hline 37 & $\begin{array}{l}\text { When I am reading an interesting story or novel, I imagine how I would feel if the events in the story were } \\
\text { happening to me. }\end{array}$ \\
\hline 38 & When I see someone who badly needs help in an emergency, I go to pieces. \\
\hline 39 & Before criticizing somebody, I try to imagine how I would feel if I were in their place. \\
\hline
\end{tabular}

Table 2. Adapted Presence Questionnaire ${ }^{2}$

\begin{tabular}{|c|c|}
\hline & Question \\
\hline 1 & How much were you able to control events? \\
\hline 2 & How responsive was the environment to actions that you initiated or performed? \\
\hline 3 & How natural did your interactions with the environment seem? \\
\hline 4 & How much did the visual aspects of the environment involve you? \\
\hline 5 & How natural was the mechanism which controlled movement through the environment? \\
\hline 6 & How compelling was your sense of objects moving through space? \\
\hline 7 & How much did your experiences in the virtual environment seem consistent with your real world experiences? \\
\hline 8 & Were you able to anticipate what would happen next in response to the actions that you performed? \\
\hline 9 & How completely were you able to actively survey or search the environment using vision? \\
\hline 10 & How compelling was your sense of moving around inside the virtual environment? \\
\hline 11 & How closely were you able to examine objects? \\
\hline 12 & How well could you examine objects from multiple viewpoints? \\
\hline 13 & How involved were you in the virtual environment experience? \\
\hline 14 & How much delay did you experience between your actions and expected outcomes? \\
\hline 15 & How quickly did you adjust to the virtual environment experience? \\
\hline 16 & How proficient in moving and interacting with the virtual environment did you feel at the end of the experience? \\
\hline 17 & $\begin{array}{l}\text { How much did the visual display quality interfere or distract you from performing assigned tasks or required } \\
\text { activities? }\end{array}$ \\
\hline 18 & How much did the control devices interfere with the performance of assigned tasks or with other activities? \\
\hline 19 & $\begin{array}{l}\text { How well could you concentrate on the assigned tasks or required activities rather than on the mechanisms used } \\
\text { to perform those tasks or activities? }\end{array}$ \\
\hline 20 & How much did the auditory aspects of the environment involve you? \\
\hline
\end{tabular}


Table 3. Custom Questionnaire (post activity)

\begin{tabular}{|l|l|}
\hline & \multicolumn{1}{|c|}{ Question } \\
\hline 1 & In the next 2 weeks I will search for more information about dyslexia or reading disabilities. \\
\hline 2 & To what extent did you feel ownership of the virtual body of the child? \\
\hline 3 & To what extent did the ethnicity of the parent figure distract or interfere with the experience? \\
\hline 4 & To what extent did your emotions mirror the child's? \\
\hline 5 & To what extent did the perceived gender of the child distract or interfere with the experience? \\
\hline 6 & $\begin{array}{l}\text { If the opportunity arose in the next 2 weeks, I may choose to donate to a charity focused on dyslexia or reading } \\
\text { disabilities. }\end{array}$ \\
\hline 7 & To what extent was the parent relatable? \\
\hline 8 & To what extent was the child relatable? \\
\hline 9 & How empathetic did you feel towards the child's struggles? \\
\hline 10 & $\begin{array}{l}\text { We are writing proposals for funding future research in our lab. In your opinion, based on the options below what } \\
\text { would be the most important topic to focus on in our research? }\end{array}$ \\
\hline 11 & Did you feel any discomfort during the experience ? (if yes, please explain) \\
\hline 12 & $\begin{array}{l}\text { Did you feel any issues with seeing or hearing things during the experience ? (if yes, how much of a problem was } \\
\text { that ?) }\end{array}$ \\
\hline 13 & $\begin{array}{l}\text { We are deciding the focus of our lab over the next 2 years on specific topics related to children's literacy. In your } \\
\text { opinion, what are the most important activities to focus on? }\end{array}$ \\
\hline
\end{tabular}




\section{APPENDIX B: INTERVIEW QUESTIONS}

\section{Table 4. Interview questions}

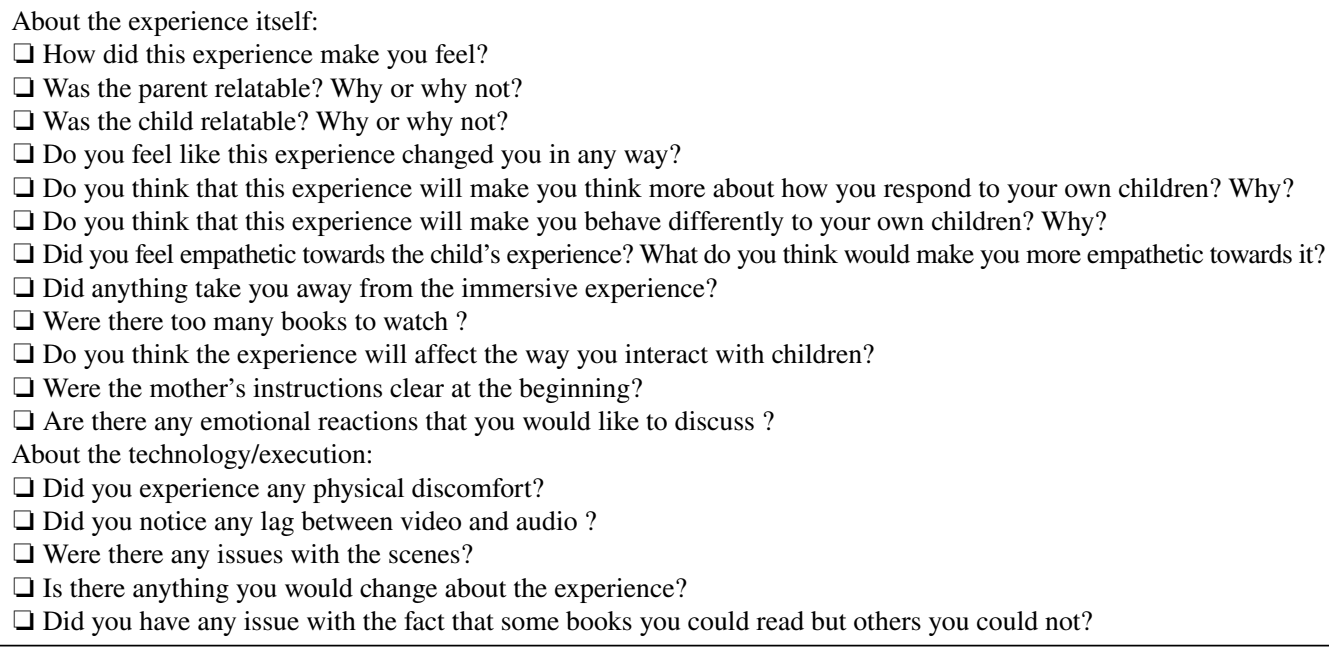

Iulian Radu is a Research Scientist at Harvard University's Graduate School of Education. His work intersects educational innovation and learner-centered design, specifically focusing on aspects of AR/VR learning, software / hardware engineering, and embodied cognition. At Harvard University, he has been researching AR for collaborative education in maker spaces (part of HGSE Learning, Innovation and Technology Lab), and VR for bias and empathy training (part of HGSE Immersion Lab). He holds a PhD from Georgia Institute of Technology focused on augmented reality for K-12 education, and has worked for international organizations creating emerging learning technologies.

Chris Dede is the Timothy E. Wirth Professor in Learning Technologies at Harvard's Graduate School of Education. His fields of scholarship include emerging technologies, policy, and leadership. In 2007, he was honored by Harvard University as an outstanding teacher, and in 2011 he was named a Fellow of the American Educational Research Association. His co-edited books include: Scaling Up Success: Lessons Learned from Technology-based Educational Improvement; Digital Teaching Platforms: Customizing Classroom Learning for Each Student; Teacher Learning in the Digital Age: Online Professional Development in STEM Education; Virtual, Augmented, and Mixed Realities in Education; Learning engineering for online education: Theoretical contexts and design-based examples; and The 60-Year Curriculum: New Models for Lifelong Learning in the Digital Economy.

Mohamed Raouf Seyam received his Ed.M in 2019 from Harvard University - Graduate School of Education. Raouf was a research assistant Harvard and MIT, and an intern at CSAIL. Raouf's research interests focus on immersion (e.g. AR/VR in education), educational data mining and collaborative learning environments (e.g. makerspaces). Further, Raouf is passionate about researching ways to predict learner outcomes based on sensor generated data.

Tianyi (Diana) Feng is a PhD in Education student at Harvard University. She holds an Ed.M from the Harvard Graduate School of Education, as well as a B.S. in Biology and a B.A. in Cognitive Science from Case Western Reserve University. Her research interests are in computer-supported collaborative learning, computer-mediated communication, and multimodal learning analytics.

Michelle Chung received a B.A. in Psychology with a minor in Education Studies from Wellesley College in 2016, and an Ed.M. in Language \& Literacy from the Harvard Graduate School of Education in 2019. 